\title{
Validation of a brief screening tool to assess adequacy of carbohydrate and fluid intakes in sports people
}

\author{
A. Castellanos and H. D. McCarthy \\ School of Human Sciences, London Metropolitan University, London, UK
}

\begin{abstract}
A low carbohydrate intake and poor hydration are major causes of premature fatigue and poor exercise performance in sports people ${ }^{(1)}$. Assessing the adequacy of intake of these dietary components can be time consuming and may require the need to undertake a weighed dietary assessment together with the use of food composition tables. A simple, self-administered tool could improve this situation, as nutrition screening tools aim to identify individuals who may be at risk for malnutrition ${ }^{(2)}$. However their use has not yet entered into sports and exercise nutrition practice. The aim of this pilot study was too develop a screening tool to assess the risk of low carbohydrate intake and poor hydration in sports people, and validate against a criterion method.

A single-sided questionnaire comprising 13 questions focussing on the intake of carbohydrate-rich foods and 5 focussing on fluid intake was developed. The carbohydrate questions focussed upon servings of starchy staples, other vegetables, sugar-rich foods, breakfast cereals, breads, fruits, pulses, confectionary, juices, sports and other soft drinks. The hydration questions addressed types, volume and frequency of drinks, thirst and urine colour. Each question had 2-3 possible answer options, scored between zero and 2. A total score between 0-6 indicated low carbohydrate intake, 7-10 at risk of low carbohydrate intake and 11 and above no risk of low intake. For hydration, an overall score of 1-2 suggested poor hydration, 3-4 at risk of poor hydration and 5-6 low risk of poor hydration. A sample of 32 participants who routinely engaged in sports and exercise were recruited to validate the tool against the calculated carbohydrate and fluid intakes from the mean of three random 24-hour dietary recalls. Carbohydrate intakes were expressed in g/d, \% of metabolizable energy intake and $\mathrm{g} / \mathrm{kg}^{-1}$ body weight/d and then ranked and divided into quartiles. Screening scores were also ranked and divided into quartiles. Spearman's correlation coefficient was used to compare rankings between methods and the percentage of subjects correctly classified into each quartile by the screening tool was calculated.

Moderate correlations between $0.410-0.514$ ( $p$ ranging between $<0.05$ and $<0.01$ ) were found for the carbohydrate intake variables. Between 50 and $100 \%$ of subjects were correctly classified into the first quartile, with values for the other quartiles ranging between $25 \%$ and $75 \%$ correct, depending on how carbohydrate intake was expressed. By far the majority of those misclassified were classified into the adjacent quartile, with $12.5 \%-25 \%$ being classified beyond an adjacent quartile. Correlations of $0.443(p<0.05)$ for fluid intake $(\mathrm{ml} / \mathrm{d})$ and $0.483(p<0.01)$ for drinking occasions were observed against the $24 \mathrm{~h}$ recall data.

These preliminary findings show that the screening tool performed reasonably well in this pilot study, with the majority of subjects being correctly classified based on the screening score. The small number of individuals who were seriously misclassified consumed sources of carbohydrate (predominantly protein shakes/supplements) that were not captured by the tool. Additionally, the small sample size exaggerated the extent of misclassification. Future work requires this tool to be modified to cover overlooked additional significant sources of carbohydrate in the diet and revalidated in a larger and more diverse sample, together with a weighed dietary intake used as the criterion method.
\end{abstract}

1. Maughan RJ \& Shirreffs SM (2011). J Sports Sci, 29, sup1.

2. Elia M, Stratton RJ (2012). Nutrition. 28, 477-94. 